\title{
An update on zika virus: history, emergence and future aspects
}

\begin{abstract}
At present time Zika virus is a serious emerging health problem worldwide. At first it was assumed to be an endemic disease but emergence beyond borders of Africa made it a serious issue globally. Zika virus was first time discovered in 1947 from the blood of Rhesus monkey in Uganda. The disease is mild during the first few days of infection with rash, fever, conjunctivitis and arthralgia. The transmission of virus occurs through the bite of an infected Aedes species mosquito but reports have also shown evidence of transmission through sexual and parental means. Till now no proper vaccines are developed for Zika virus. Zika Virus after being studied for so long for its pathology, virology, vectors, genetic diversities and its ability to co-exist with other viral strains; is still a big question for its transmission factors, viral strains, their adaptability and treatment. This review of literature was carried out to spread awareness among the general public for adversity and thus prevention of this disease globally.
\end{abstract}

Keywords: aedes; endemic; health issues; modes of transmission; public awareness; virus
Volume 4 Issue 2 - 2019

\author{
Muhammad Khan,' Rabia Khan,' Sarmir \\ Khan, ${ }^{2}$ Sohail Ahmad Jan, ${ }^{3}$ Azhar Hussain \\ Shah, ${ }^{3}$ Tauseef Ahmad ${ }^{4}$ \\ 'Centre for Human Genetic, Hazara University Mansehra, \\ Khyber Pakhtunkhwa, Pakistan \\ 2Department of Genetics, Center for Human Genetics, Hazara \\ University Mansehra, Khyber Pakhtunkhwa, Pakistan \\ ${ }^{3}$ Department of Biotechnology, Hazara University Mansehra, \\ Khyber Pakhtunkhwa, Pakistan \\ ${ }^{4}$ Department of Epidemiology and Health Statistics, Southeast \\ University Nanjing, China
}

\author{
Correspondence: Sarmir Khan, Department of Genetics, \\ Center for Human Genetics, Hazara University Mansehra, \\ Khyber Pakhtunkhwa, Pakistan, \\ Email sarmirkhan999@gmail.com
}

Received: April 15, 2019| Published: May 30, 2019

Abbreviations: A, aedes; $\mathrm{CDC}$, centers for disease control and prevention; EEE, eastern equine encephalitis; JEV, japanese encephalitis virus; PAHO, pan american health organization; PHEIC public health emergency of international concern; WNV, west nile virus; SLEV, st. louis encephalitis virus; WEE, western equine encephalitis virus; VEEV, venezuelan equine encephalitis virus; ZIKV, zika virus

\section{Introduction}

It was a story of 1947, that Zika forest of Uganda had rhesus macaque with Zika virus in its blood. Virus was introduced 70Years later in Brazil through Pacific Islands and gradually emerged all over America within a year. Since 1953 to 2007 only 13 naturally acquired cases were registered. During this time period the state of Yap and Federated State of Micronesia had major outbreak of virus among population of 6700 people with viral infection in 5000 of them. In 2013 to 2014 French Polynesia had 32000 victims of Zika virus. Growing evidences have associated Zika virus (ZIKV) infection with congenital malformations, including microcephaly. ${ }^{1}$ Brazil had 38 infants born with microcephaly in September 2015 in the areas of first outbreaks of virus, later it exceeded to 4300 cases of microcephaly in 2016. New evidence supports the transmission of virus through parental and sexual means other than mosquito (Adeshensilli) bites (A. Agypti and A. albopictus). Strains with Asian genotype have to be found in America which show close association with strains from Yap, Cambodia, Thailand, and French Polynesia. In 2007, a clinically distinct disease from dengue, with symptoms of rash, arthralgia and conjunctivitis, was identified by physicians with 49 confirmed and 59 probable cases of illness. The illness was named to be Zika virus on the basis of Zika RNA strain. Mosquito responsible for spread of virus was proved to be Adeshensilli. ${ }^{2}$ First outbreak recorded in Asia and Africa was on Yap Island in the southwestern Pacific on April 2007. After the Yap outbreak in 2007 viral transmission was stated to be occurring in French Polynesia with 29000 case studies in October 2013. The viral strain in Polynesia was said to have Asian lineage showing similarities to those from Yap State and Cambodia. One Patient with acute Zika virus infection after a week developed Guillaine-Barre Syndrome (GBS) in November, however most of patients showed mild symptoms of infection in start. FP population started to have incident rates of 1-2/100,000 members of population every year. Outbreaks of Zika virus occurred in New Caledonia, the Cook Islands, and Easter Island. ${ }^{3}$ A German traveler returning from Thailand was confirmed to transport Zika virus into Europe in November 2013 and showed symptoms of viral infection as arthralgia, per articular swelling, diffuse maculopapular rash, malaise, fever and chills after 12 days of his return to home. Laboratory tests confirmed the presence of IgM positive and IgG negative for Dengue infection while IgM and IgG confirmation for Zika alone with viral RNA presence through RT-PCR. ${ }^{4}$

Zika's relation with neurological problems as GBS and microcephaly made it a concern in America based on its geographic spread. Brazil had its first confirmed Zika case in March 2015 since then 2782 cases of microcephaly were confirmed only in Brazil. Timeline: 1947 - ZIKV first isolated from Rhesus monkey in Uganda. ${ }^{5}$ In 2007: First major Zika outbreak in Yap islands of Micronesia ${ }^{6}$ 2013: Bigger outbreak in French Polynesian islands. ${ }^{7}$ February 2014: First appearance in Western Hemisphere (Chile) (Petersen et al., 2016a). March 2015: Major outbreak in Brazil, rapid spread to Americas. ${ }^{5}$ February 1, 2016: WHO declared Zika virus disease as a Public Health Emergency of International Concern (PHEIC). ${ }^{8}$ First report of Zika virus (ZIKV) in Brazil: Early 2015, in 8patients with rash, fever, conjunctivitis and arthralgia, they had RT-PCR positive for ZIKV growing since 2007. ${ }^{9}$ First outbreak of ZIKV in the state of Rio de Janeiro was reported through doubted $(n=364)$ and laboratory confirmed cases $(\mathrm{n}=119)$. During January and July 2015 clinical trials gave 364 suspected cases of ZIKV. 119(45.4\%) had their RT-PCR positive for ZIKV RNA. All cases had strains showing Asian lineage of virus. Myalgia, lower back pain, Headache, non- 
purulent conjunctivitis and arthralgia steady with the case meaning of suspected ZIKV illness had such signs issued by the Pan American Health Organization (PAHO). Be that as it may, fever, when present, was low power and short-termed. Authors suggested that fever is not that much of a concern here but pruritus should be second significant clinical sign. ${ }^{10}$

\section{Transmission of Zika virus and its lethal effects}

ZIKV shows its endemic nature, transmission and co-occurrence of neutralizing antibody with that of DENV and CHIKV. ZIKV is of Japanese encephalitis virus (JEV) sero-complex origin thus it has tendency to cross react and cross neutralize antibodies for this group of viruses as DENV. Zika infection is a disease caused by a group of viruses spread in people by the bite of infected arthropods or a type of insect such as mosquito and tick. Pathogen in human is not habitual or common because it can pass on or shed for year in the semen. ${ }^{11}$ Zika virus (ZIKV) is a mosquito-borne virus that has garnered a lot of attention in recentYears, due to its multiple neurological defects. ${ }^{12}$ Nine Ades mosquito species and anopheles mosquito are successful hosts of ZIKV. Emergence of A.albopictus in more temperate regions gives an efficient expansion. ZIKV spread is more complex than DENV and CHIKV because of its RNA being present in saliva and urine samples of patients making ZIKAV transmission through parental and sexual means. Emergence of ZIKV and microcephaly coincided with each other. ${ }^{13}$ The general wellbeing reaction incorporates expanded ability to test for Zika infection, preventing disease in pregnant ladies, observing diseased pregnant ladies and their baby for unfavorable results, controlling mosquitoes, and guaranteeing the security of blood items. In a joint effort with PRDH and CDC a locally established vector control program concentrated on pregnant ladies is in progress. Ladies are reached through WIC centers, and are offered source decrease facilities (e.g., eviction of water holders that can fill in as mosquito reproducing destinations), larvicide application, and indoor and open residual spraying utilizing deltamethrin. ${ }^{14}$ Exploring the introduction of virus to America, the phylogenetic, epidemiological and mobility data for quantification of ZIKV was recorded. From Samples collected during the spread of disease nine coding regions of virus were sequenced through Next Generation Sequencing. Samples were collected from a diseased newborn with inborn deformities and microcephaly in Ceara and one from a deadly grown-up case with rheumatoid and lupus disease from Maranhao state. All viruses sampled in America when sequenced appeared to have monophyletic cluster within the Asian lineage. ZIKV emergence in Brazil had coincided with air travelers from endemic areas for ZIKV as Pacific Islands. Reports suggested that cases of microcephaly correlated with ZIKV in 17 th week of pregnancy, although this correlation can't show causation. ${ }^{15}$

A. albopictus or A.Agypti was considered "present" in a state in a given schedule year if somewhere around one specimen of any life phase of the mosquito was gathered, utilizing any collection technique, during that year; areas were additionally arranged dependent on whether species types was gathered in 1,2, or at least 3 Years, with no refinement of whether gatheringYears were back to back. Between January 1, 1995 and December 31, 1999, occurrence of A. Agypti was reported from 11 provinces in Arizona, Texas, and Florida. In 2004, A. Agypti was reported from 80 countries and records were included along the southern level just as sporadically along the East coast. Generally speaking, between January 1995 and
March 2016, 183 areas from 26 states and the District of Columbia detailed event of A. Agypti, and 1,241 regions from 40 states and the District of Columbia revealed event of $A$. albopictus. What's more, $A$. Agypti was collected in at least 3 Years from 94 areas from 14 states and the District of Columbia, and A. albopictus was gathered amid at least 3 Years from 514 provinces in 34 states and the District of Columbia. The authors concluded that there is a need for systematic surveillance of A. Agypti and A. albopictus in the US. ${ }^{16}$ Yap state, Federated state, Micronesia, Southeast Asia and Western Pacific had viral outbreak in 2007.First local transmission in America and Brazil occurred during May 2015. By January 2016 virus emerged in further 19 countries. Zika virus can lead to microcephaly and fetal losses (1) Mosquito-to-human, (2) intrauterine, (3) intrapartum, (4) sexual, (5) blood transfusion, and (6) laboratory exposure are means of transmission. Imported rate of transmission increase in US may lead to local transmission of diseases. ${ }^{17}$ Ades mosquito has higher rates to the transmission of virus through travels internationally. Prenatal transmission and sexual transmission through semen are not that common. There has been reported change of the human Zika virus infection during its geographical expansion- from endemic, mosquito borne infection causing mild illness from Africa to Asia; from 2007 onward there were large outbreaks; from 2013 outbreaks linked to GBS and microcephaly from pacific region to the Americas. ${ }^{18}$ Brazil had Zika cases in 2014 and 2015 and now 2015 is time for Columbia. The diffuse or localized rash and non-secreting conjunctivitis are the most common and distinguishing clinical features. Conjunctivitis usually lasts 5 to 6 days. Other features include joint pain and swelling, fever (mild), general malaise but disease is not as severe as dengue fever. Brazil is working its way to study sings of acute phase of disease along with the long-term complications. Lab tests in Columbia are carried out to distinguish measles from DENV and CHIKV. Preventive measures include destroying potential mosquito breeding sites. Video of clinical signs, such as maculopapular rash and conjunctivitis were shown. ${ }^{19}$ A 21 Years old woman was first case of Zika in Cuba. First case in Dominica was of an individual having no travel history. Researchers have guessed that Ades mosquito would be spreading around US from NYC to LA during peak summer seasons and so is Zika's risk expected during the season. Travel patterns predict the next hot spots for Zika as Southern Florida and improvised parts of South Texas. There were 854 cases of microcephaly reported in Brazil at the time of this publication. ${ }^{20}$ Total of 233 studies were analyzed which were published since 2011 and were then surveyed in March 1, 2016. Many studies regarding epidemiology, pathogenesis and molecular evaluation were based on observational study design. Complications related to human infection, transmission sources, pathology and human burdens with ZIKV had certain knowledge gaps. Zika Virus has similar epidemiological plus transmission patterns and clinical associations being an arthropod-borne virus to those of CHIKV and DENV. Spread of virus globally signifies it as DENV and CHIKV. ${ }^{21}$

This review considers the utilized serological and observation information to assess the likelihood of disease with Zika infection for every seven day epidemic of the scourge utilizing medical records to distinguish all instances of microcephaly from September, 2013, to July, 2015. A sum of 8 instances of microcephaly was identified (88\%) happened in the 4-month time span March 1 to July 10, 2014. Of the six times of danger during pregnancy, four clarified the planning of instances of microcephaly essentially superior to anything the no affiliations demonstrate. The two that showed improvement over 
the no affiliation display accepted the time of hazard was confined to trimester three or trimesters two and three. The planning of these cases was best clarified by a time of hazard in the first trimester of pregnancy where the standard predominance of microcephaly was two cases $(95 \%$ CI $0-8)$ per 10,000 neonates, and the danger of microcephaly connected with Zika infection was 95 cases (34191) per 10,000 ladies diseased in the primary trimester. Increased microcephaly was a case in other trimesters but first trimester had no support from data. ${ }^{22}$ There was no noted greater risk of infection in pregnant vs non-pregnant; common symptoms consisted of fever, rash, and arthralgia; GBS only reported severe maternal morbidity; fetal/newborn complications included fetal and infant neurological and visual deformities from the norm; fetal development confinement, stillbirth and perinatal death, with microcephaly the man neurological problem reported in 8 studies. Some studies reported not maternal symptoms during infection and some studies reported normal birth outcomes. ${ }^{23}$ Some woman during the 11th week of gestation got infected while visiting Mexico, Guatemala, and Belize. Serologic examination performed a month after the indications of sickness and was on an outing to her local Finland was positive for IgG antibodies and negative for IgM antibodies against dengue infection. Resulting serologic investigation was positive for both $\operatorname{IgG}$ and $\operatorname{IgM}$ antibodies against ZIKV. Serologic examination for the presence of chikungunya virus was negative. Noted decline in the fetal head outline from the 47 th percentile at about four months to the 24th percentile at 20 weeks, alongside an abnormal intracranial ultrasound and MRI at 20 weeks gestation. Pregnancy was ended at 21 weeks of gestation. The authors along these lines recommend the utilization of quantitative RT-PCR strategies for analytic methodology for continuous placental or fetal infection in pregnant ladies. ${ }^{24}$

\section{Why understanding Zilka was a complication?}

During Dengue and Zika's co-epidemic, a French Polynesian woman developed conjunctivitis, coetaneous rash, febricula and myalgia and after 7 days was treated with GBS. Laboratory tests as RT-PCR was negative for ZIKV during first 8 days of influenza like symptoms but later on from 8th to 28th day's samples gave positive results for IgM specific ZIKV and IgG specific DENV. ${ }^{25}$ French Polynesia during Zika outbreak time period October 2013 and April 2014 had almost 42 patients diagnosed with GBS. Two control groups were observed for developing relationship of Zika to GBS. First control group had $n=98$ with no febrile illness, had sex, age and residence matched patients. Second control had $n=70$ with no neurological disorder but had age matched and acute ZIKV.98\% of 42 patients diagnosed to have GBS had threatening to Zika IgM or IgG, and $100 \%$ had killing antibodies versus $56 \%$ of 98 in control group $1.39(93 \%)$ patients with GBS had Zika virus IgM and $37(88 \%)$ had transient affliction in a median of 6 days before start of neurological indications; rash, arthralgia and fever were the most commonly reported symptoms. Every 1000 ZIKV carriers in population had chance to develop GBS rate as 0-24patients with no reported deaths. Respiratory assistance was required for 12 patients. $31 \%$ patients had anti-glycolipid antibody action. Only one case of GBS had plasmapheresis, rest had immunoglobulin treatment. Typical antiganglioside AMAN antibodies were rarely found. ${ }^{26} \mathrm{~A}$ 15year old female showed arthralgia, rash, abdominal pain, retro-ocular pain and fever for four days, had no history for any acute chest problem. Patient was admitted to severe intensive care unit as she developed worsened respiratory tract problems. She was initially diagnosed with DENV but RT-PCR confirmed her ZIKV attack. Patient died after 37 hours of initial presentation along with intensive cure and preventions. Functional asplenia with drepanocytes and splenic sequestration, erythro-phagocytsis of kupferrv cells were represented through Autopsy. ${ }^{27}$ After the emergence of ZIKV in Brazil authors reported ophthalmic findings in three infants with microcephaly. All of these three infants had unilateral ocular problems and cerebral calcification. Mothers had no symptoms during gestation period but one mother had developed rashes and arthralgia during first trimester. Reflexloss, neuro-retinal atrophy and macular pigment mottling were observed in ocular results. $^{28}$

\section{Were laboratory findings of any assistance?}

RT-PCR, phylogenetic analysis, nucleic acid testing and serological test reports from 2007 Zika emergence in Yap state and Federated state of Polynesia were reported. Antibody testing results lead specimens into primary and secondary infections. Serologic analysis was performed for IgG and IgM and PRNTs of all acuteand recovering stage matched specimens got during the widespread Yap Island specimens had secondary flavivirus contagions. Serologic information demonstrated that ZIKV-infected patients can be positive in an IgM examine for DENVs, especially if ZIKV is a secondary flavivirus disease. On the off chance that ZIKV is the primary flavivirus experienced, then cross-reactivity was found to be minimal. The PRNT result from one patient suggested previous yellow fever vaccination. DNA sequence and phylogenetic analysis was performed of RT-PCR positive specimens. Real-time RT-PCR of 157 specimens was also performed, of which 17 tested positive, 10 were equivocal, and 130 tested negative. Most (15 of 17) of the ZIKV-positive samples were from specimens gathered $<3$ days after infection beginning. ${ }^{29}$ In Japan from 2006-2008, 52 Dengue patient's serum and urine samples were tested. Day 6-16 had shown 50\% and above Dengue viral infection for urine samples. ${ }^{21}$ Urine and serum samples had similar nucleotide sequences through PCR outcomes. RT-PCR can act as reciprocal test for convalescent stages of Dengue infections.$^{30}$ Serum and urine samples were obtained from 6 patients who showed the same symptoms of cutaneous maculopapular rash of the trunk and extremities, and mild leukopenia and thrombocytopenia. ZIKAV RNA was detected in all 6 urine samples. For all cases with consecutive specimens, ZIKAV RNA was noticed in the urine $\leq 15$ days (run 10days to $>20$ days) after beginning of indications, which was $>7$ days after it was not notice in serum tests; In serum samples, ZIKV was identified in patient serum until a rash was watched/days 2-3 after illness beginning. ${ }^{31}$ During the French Polynesian Zika Outbreak, from October 2013 to March 2014, a few patients saw to give manifestations of intense stage Zika fever were tried negative in blood by ZIKV continuous PCR (RT-PCR). Utilization of saliva was considered as an alternate sample for routine ZIKV RNA detection. Over a few months time span 1,067samples (saliva just, blood just, or the two samples) were collected from 855patients showing signs of Zika sickness. ZIKV was all the more often detected in saliva contrasted with blood. For the 182patients with the two samples collected, tests were positive for $35(19.2 \%)$ in saliva while negative in blood and tests were positive for $16(8.8 \%)$ in blood while negative in saliva.

\section{Conclusion}

The utilization of saliva test expanded the rate of molecular identification of ZIKV at the intense period of the sickness yet did not develop the window of recognition of ZIKV RNA. Saliva was 
quite compelling when blood was hard to collect (kids and neonates particularly). ${ }^{32} \mathrm{~A}$ total of six Israeli travelers with symptoms of ZVD had serum, whole blood and urine samples examined; follow-up serum, urine and whole blood specimens were only available for five patients. QRT-PCR was positive for only one of six serum samples, which was drawn 3 days post-symptom onset. The negative 5 serum samples were drawn 5days post-symptom onset. Of five samples tested for Zika virus IgM, four were IgM positive, including three, which were additionally IgG positive. Urine was positive in three samples collected from three patients 5 to 26days post-manifestation beginning; a second urine samples collected 46 days after beginning was negative. Generally, entire blood samples were positive for as late as 2 months (58 days) post-side effect beginning, which was longer than for urine (26 days) and serum (3 days). The authors propose that these discoveries recommend the utility of entire blood in Zika contagion diagnosis. ${ }^{33}$

\section{What are means of viral transmission?}

Reviews show that these viruses have tendency to cause congenital neurological abnormalities; Japanese Encephalitis Virus (JEV), West Nile Virus (WNV), St. Louis Encephalitis Virus (SLEV), Western Equine Encephalitis virus (WEE), Venezuelan Equine Encephalitis Virus (VEEV), and Eastern Equine Encephalitis (EEE) virus. ${ }^{34}$ ZIKV can cause devastating congenital Zika syndromes in pregnant women and Guillain-Barre syndrome in adults. ${ }^{35}$ Possible ways of perinatal transmission through two reported cases were said to be delivery, breast-feeding, Tran's placental and close contact as saliva. ${ }^{36}$ Newborn from mother\#1 stayed healthy for 5days post-delivery, mother delivered the risk during delivery, was probably viremic before and during delivery. Mother\#2 had complications during delivery, cesarean was performed; pregnancy complicated by gestational diabetes and intrauterine growth restriction, newborn developed rash post 3rd day of delivery, had severe hypotrophy, 8/9/9 Apgar and jaundice while although mother was incubating during delivery. Infant's serum was positive 4days post-delivery and serum from mothers was positive on 2nd day post-delivery. Both newborns had similar RNA loads but newborn\#1 showed no symptoms. Newborn \#2 displayed low platelet count and elevated total bilirubin on day3. Breast milk samples from both mothers positive for ZIKAV; however, cell culture was negative for replicative ZIKAV. There is no firm data from RT-PCR, to prove trans-placentals. But data reported gave clear evidence for transmission through close contact and breast milk. ${ }^{37}$ Complications from viral infections (i.e., preterm labor) in pregnancy supposed to be result of subsequent polymicrobial infections- viral infection of the placenta can affect the normal interaction with local bacteria, causing a pro-inflammatory "cytokine storm" which causes preterm delivery. Murine model showed; in which pregnant mice were inoculated with murine herpes virus. All mice delivered preterm; significant inflammatory responses were also noted in the placenta and deciduain addition to reported adverse fetal outcomes. ${ }^{38}$ Investigations were done about the transmission of ZIKV in primary human trophoblast cells. Studies showed that PHTS are refractory to the two strains of ZIKV for attack; one is of Asian lineage and other one from African lineage with both strains having $99 \%$ similarity to strains found in Brazil. ${ }^{39}$ The constitutive release of the type III IFN IFN11 from Conditioned medium taken from PHT cells can help protect non-trophoblast cells from attack of Zika. The release of anti-viral type III interferon's from primary human trophoblasts make them refractory to ZIKV infection which led authors to suggest that ZIKV uses alternate means to affect placenta rather than directly infecting it. ${ }^{40}$
RT-PCR for urine and blood samples was used to test 88 women who developed rashes in previous 5days. Mild viral infection from $5-38$ weeks of gestation of the 88 ladies, $72(82 \%)$ tested positive in one or the two samples; 60ladies had positive serum specimens; 46 had positive urines specimens; 34 had positive samples in the two specimens; 12 had positive outcomes in urine samples only, and 26 had positive outcomes in blood specimens only clinical features were typical of Zika virus infection. Among positive ladies, the greater part detailed comparable sicknesses in other relatives, and $21 \%$ revealed that their accomplice had been sick. The greater part the ladies gave intense infection in the second trimester of pregnancy. 2 positive ladies prematurely miscarried during the first trimester. Fetal ultrasound was performed in 42 positive ladies; fetal variations from the norm were distinguished in 12 of the positive women. 5 of the 12 embryos had intrauterine development restriction, with or without associated with microcephaly; 4 fetuses had cerebral calcifications and other CNS alteration in 2 fetuses; abnormal arterial flow in the cerebral or umbilical arteries was seen in 4 fetuses; oligohydramnios and anhydramnios were seen in 2fetuses; two fetal deaths occurred in two cases after 30 weeks gestation. At the time of this report, 8 of the women who received ultrasound have provided (6 live and 2 stillbirths). ${ }^{41}$

In Brazil, a sum of 1501 newborn children conceived between November 19, 2015 and February 27, 2016 got full examinations. Suspected cases included live infants with microcephaly characterized as $33 \mathrm{~cm}$ or less for term young men and young ladies, which was decreased to $32 \mathrm{~cm}$ on Dec 12. There were 5 categories (1) definite, or newborns with evidence of Zika infection during pregnancy via PCR or serology, independent of other findings; the other 4 categories included newborns without laboratory evidence: (2) highly probable, or newborns with specific imaging reports and negative for syphilis, toxoplasmosis, and cytomegalovirus; (3) moderately probable, or newborns with specific imaging reports but positive for one or more of the three infections; (4) fairly plausible, or infants with imaging reports coming up short on a definite depiction of the findings, for which a state-level doctor concluded that an inherent disease was likely involved, for whom research facility results for the three infection were negative or inaccessible; (5) discarded of cases, or newborns not included in any of the cases. 899 cases were discarded; of the remaining 602 cases, 76 were definite, 54 highly probable, 181 moderately probable, and 291 somewhat probable of congenital Zika virus syndrome. $^{42}$

Epidemic struck its peak during November, 2015. The authors conclude that Zika virus congenital syndrome is a new teratogenic disease which must be detected with revised screening criteria given that many cases (definite and probable) present normal head circumference values and there is no report of rash with the mother. ${ }^{43}$

In excess of 4,700 associated cases with microcephaly were accounted for from mid-2015 through January 2016, albeit extra examinations may in the long run outcome in a changed lower number. December 2015,formalin-fixed, paraffin-embedded (FFPE) tissues samples from two babies with microcephaly (born at 36 and 38 weeks gestation) who dead within 20 hours of birth, and two miscarriages (fetal losses at 11 and 13 weeks) were submitted to CDC, from Rio Grande do Norte in Brazil. Each of the four moms had fever, rash, during the first trimester of pregnancy; all were negative for clinical signs of active infection delivery or miscarriage. Sample from every one of the four cases were positive for RT-PCR. 
In infants just cerebrum tissue was positive for RT-PCR. Placental tissue from one premature delivery demonstrated heterogeneous chorionic villi with calcification; chorionic villi sampling was normal for the other miscarriage viral antigen was noted in mononuclear cells (presumed to be glial cells and neurons inside the mind) of one infant, and inside the chorionic villi from one of the miscarriages. It is concluded that cerebrum and early gestational placental tissue may be the favored tissue for after death viral analysis. Non-frozen, formalin-fixed specimens or FFPE blocks are the favored sample type for histopathology assessment and immune histo-chemistry. RT-PCR can be performed on either fresh frozen or formalin-fixed specimens. ${ }^{44}$

On January 19, 2016, CDC discharged between time rules suggesting that pregnant ladies who had made a trip to territories with continuous neighborhood transmission of Zika infection and who had side effects steady with Zika infection ailment be tried for Zika infection disease; these were updated on February 5 to offer Zika infection testing to every pregnant lady with Zika infection exposure, paying little attention to the presence of side effects. From August 1, 2015 to February 10, 2016, the CDC got 257requests for testing of ZIKV for pregnant ladies. As of February 17, 2016, only nine of the pregnant women were determined to be positive for Zika virus infection. All reported a clinical sickness, incorporating eight ladies with $\geq 2$ signs and additionally manifestations, and one with a generalized up rash six pregnant ladies with Zika infection illness who revealed side effects during the first trimester, had the accompanying results: early pregnancy losses (2), elective pregnancy terminations (2), and conveyance of a live newborn child with microcephaly (1); one pregnancy is ongoing. In two ladies with Zika infection contamination who had manifestations amid the second trimester of pregnancy, one evidently sound newborn child has been born and one pregnancy is ongoing. One pregnant lady reported indications of Zika infection contamination in the third trimester of pregnancy, and she delivered a sound newborn child. ${ }^{45}$ Zika virus disease including microcephaly outbreak was noted in Brazil in 2015. By 28 November 2015, 646 cases had been reported in Pernambuco state, Brazil alone. Two microcephaly cases were examined: (1) in case 1 , fetal ultrasound at 30.1 gestational weeks showed head circumference (HC) 2.6 SD below expected value, brain atrophy, and course white matter calcifications. (2) In case 2, HC at 29.2 weeks' gestation was 3.1 SD below expected value, with brain atrophy, ventriculomegaly, cataracts, and intraocular calcifications. Six microcephaly cases studied were born to mothers who had Zika symptoms during pregnancy, and all cases had $\mathrm{HC}$ below the 10th percentile. Apart from ZIKV disease, other intrauterine infections that can retard fetal brain development include cytomegalovirus (CMV), toxoplasmosis, herpes virus, syphilis, rubella, and West Nile encephalitis virus (flavivirus). Among these, Zika virus related fetal brain atrophy and microcephaly closely resembles CMV and West Nile encephalitis virus disease. ${ }^{46}$ The prenatal Zika virus (ZIKV) infection caused severe microcephaly [58]. By January 2016, transmission had been affirmed in 22 of Brazil's 26 states and the government locale, and in every one of the five districts of the nation; this was a major increase from 2015, occurring largely in the Northeast region of the country where the Zika outbreak first began. In October 29th 2015, increases in the number of microcephaly cases were reported by the Brazil Ministry of Health $(\mathrm{MoH})$ as a potential Public Health Emergency of International Concern. The case definition for microcephaly changed from a head circumference of $\leq 33 \mathrm{~cm}$ to $\leq 32 \mathrm{~cm}$ in any full-term newborn; which is currently used countrywide. Directed by the $\mathrm{MoH}$ and Secretaries of Health from the influenced zones, an investigation of 574 instances of microcephaly, was recognized through a recently settled specially appointed microcephaly reconnaissance framework which identified temporal and geospatial proof connecting the occurrence of febrile rash sickness steady with Zika infection illness during the primary trimester of pregnancy with the increase birth predominance of microcephaly. The predominance of microcephaly in 15 states with research facility/RT-PCR affirmed Zika infection transmission (2.8 cases per 10,000 live births) fundamentally surpassed that in four states without affirmed Zika infection transmission $(0.6$ per 10,000). Of the 574 cases, $58.5 \%$ (336) were females. The average head circumference was $29.0 \mathrm{~cm} .{ }^{47}$ Patients with clinical symptoms of ZVD from August 9, 2015, to April 2, 2016, were included in the analysis; a subset of pregnant women was included in the analysis of pregnancy outcomes. A total of 65,726 instances of ZVD were reported in Colombia; 2485 (4\%) were affirmed by means of RT-PCR. 11, 944 pregnant ladies with ZVD were reported in Colombia, with 1484 (12\%) confirmed through RT-PCR measure. In a subgroup of 1850 pregnant ladies, over $90 \%$ of ladies diseased during the third trimester had no babies with evident deformities. From January 1, 2016, to April 28, 2016, a sum of 50 newborn children with likely microcephaly were reported to the national surveillance framework for birth defects; of these instances of microcephaly, four had lab proof of inherited ZVD; all were born to asymptomatic moms who were excluded in the ZVD surveillance framework. Authors presume that the maternal disease with the Zika infection during the third trimester of pregnancy isn't connected to basic deformities from the norm in the embryo. Though, the observing of the impact of ZVD on pregnant ladies in Colombia is continuous. ${ }^{42}$ WHO declared a Public Health Emergency of International Concern in February 2016 and in April 2016, CDC announced the presence of a causal association between pre-birth ZIKV and microcephaly alongside with other neurological abnormalities. In spite of the developing number of cases with maternal indications of disease or potentially presence of ZIKV in tissues of affected babies or embryos, the authors survey that at present it's tough to decide the extent of increase of microcephaly prevalence in Brazil, just as the effect of different factors causing congenital neurological conditions. ${ }^{48}$ Authors describe microcephaly, infectious agents known to cause birth defects, the investigation of the causal link between Zika virus and birth defects, clinical management, counseling women of reproductive age and pregnant patients, and challenges with (lack of) data and the current outbreak. The authors suggested that new mutations in the Zika virus could affect viral replication in humans, leading to increased virulence and therefore improved probabilities of viral transmission to extra credulous mosquito vectors. More research is needed to evaluate $\mathrm{ZIKV}$ and its effect in inborn issues.

A pregnant woman with a history of travel to ZIKV-affected country is tested for ZIKV infection if has symptoms within 2 weeks of travel. If test is positive or inconclusive, then fetal ultrasound (U/S) is performed to detect microcephaly and/or intracranial calcifications; and amniocentesis to test amniotic fluid for ZIKV is offered. If maternal ZIKV test is negative, fetal ultrasound is performed. And if microcephaly or calcifications are present, then amniotic fluid can be tested for ZIKV. If pregnant woman with history of travel has no symptoms, then fetal U/S is done to look for microcephaly/ calcifications. If either finding is present, mother is tested for ZIKV, and amniocentesis for ZIKV testing is offered. If initial U/S does not have findings, serial ultrasounds (every 3-4weeks) are performed to assess for microcephaly/calcifications. Maternal-fetal-medicine or 
infectious disease specialist is consulted for any suspected ZIKV transmission. Preventive measures: (1) avoid travel to ZIKV affected countries during pregnancy, and (2) if travel is unavoidable, then engage in mosquito bite prevention steps, such as use of permethrintreated clothing and gear. Treatment is entirely supportive: rest, fluids, antipyretics, and analgesics. Rule out Dengue (DENV) before treating with NSAIDS. Avoid NSAIDS in pregnancy. ${ }^{49}$ Females with conceivable exposure that don't dwell in a region with dynamic transmission-Women and men with likely exposure to Zika infection however without clinical disease steady with Zika infection illness should hold up something like two months after exposure to attempt conception. Men with possible exposure who do not reside in an area with active transmission- Men should wait at least 6 months after symptom onset if they have been diagnosed with Zika virus disease. Females and males with conceivable contact to Zika infection however without clinical sickness steady with Zika infection illness should hold up no less than about two months after introduction to endeavor origination. Women and men with possible exposure to Zika virus but without clinical illness consistent with Zika virus disease should wait at least 8 weeks after exposure to attempt conception. Women and men who reside in areas with active transmission women with Zika virus disease should wait at least 8 weeks from symptom onset before attempting conception; men with Zika virus disease should wait at least 6 months from symptom onset before attempting conception. Women and men who do not have clinical illness consistent with Zika virus disease and who desire pregnancy should talk with their health care providers. In regards to testing, pregnant women with possible exposure who do not reside in an area with active transmission should be tested according CDC's updated interim guidance Figure 1 pregnant women who do reside in an area with active transmission should be evaluated and tested in accordance with CDC updated interim guidance. ${ }^{50}$ To determine whether Zika virus infection during pregnancy causes these adverse outcomes, the authors evaluated data using criteria that have been proposed for the assessment of potential teratogens Shepard's criteria and Bradford Hill criteria. On the basis of this review using both criteria frameworks, it was inferred that a causal relationship exists between pre-birth Zika virus infection and microcephaly and different genuine brain abnormalities. Proof that was utilized to help this causal relationship included Zika virus infection it time during pre-birth development that were consistent with the imperfections observed; a particular, rare phenotype including microcephaly and related brain abnormalities in babies or newborn children with assumed or affirmed inherited Zika infection contamination; and information that firmly bolster biologic credibility, including the identification proof of Zika infection in the brain tissue of affected fetuses and babies. ${ }^{51}$

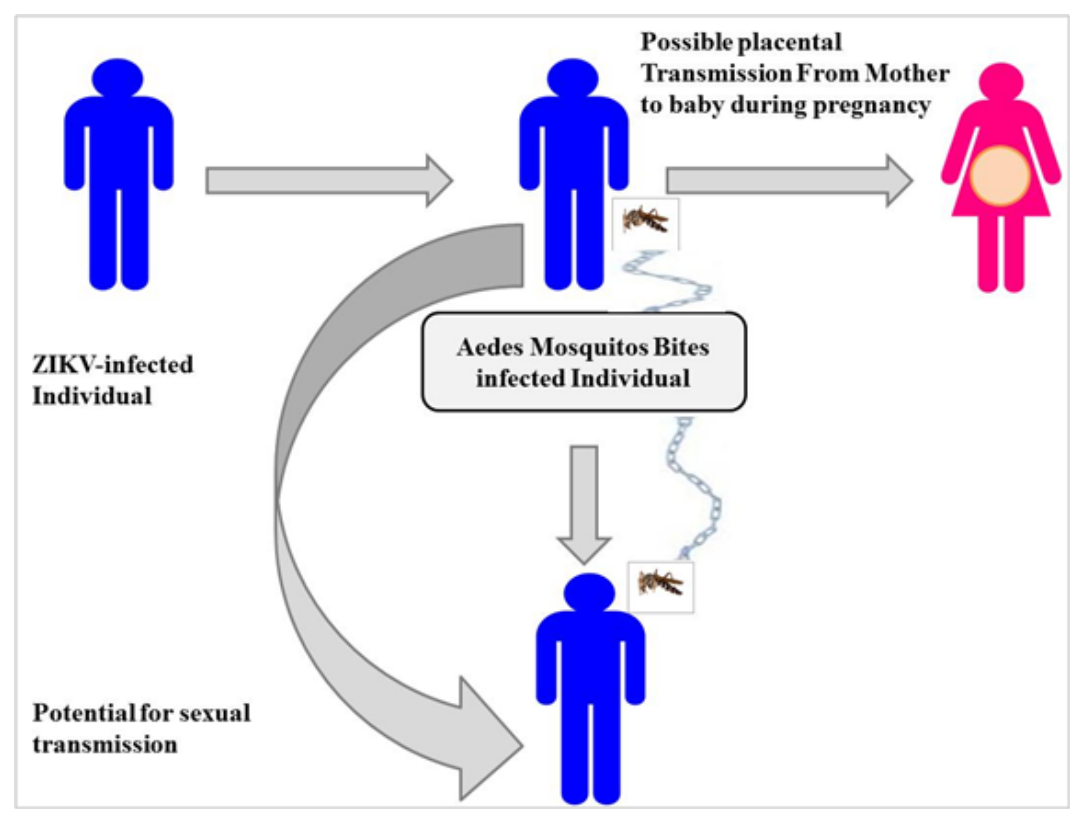

Figure I Schematic representation of Zika virus transmission, including both the traditional mosquito vector as well as the more recently described via sexual intercourse and blood transfusion. Also shown is the possibility of placental transmission of Zika virus.

During January 2016, a retrospective preliminary analysis of a cohort of 28 pregnant women with RT-PCR confirmed ZIKV infection admitted to maternity units in Sincelejo, Sucre (north, Caribbean coast region), Colombia were recruited and followed-up $21(75 \%)$ reported ZIKV symptoms during pregnancy, 7 (25\%) had no reported symptoms. Serologies were negative for chikungunya and dengue Infection occurred during first trimester for 1 (4\%), second trimester for $12(43 \%)$, and the third for $15(54 \%)$ Clinical symptoms were typical of arboviral infection (rash, fever, arthralgias, and conjunctivitis). Ultrasounds in two cases proposed brain calcifications, in one woman there was a twin pregnancy. ${ }^{52}$ First identified case of vertical WNV transmission reported during the $2002 \mathrm{WNV}$ epidemic in the US. Mother was diagnosed with WNV meningoencephalitis during pregnancy with positive serology, and the newborn infant had chorioretinitis and severe cerebral abnormalities including white matter loss, with serum and CSF positive for WNV antibodies. ${ }^{53}$ WNV-specific antibodies in cord blood from baby deliveries were estimated to decide the frequencies of WNV infections during pregnancy and of intrauterine WNV diseases. Umbilical cord blood was collected from 549 newborn children and screened for WNV IgM and IgGantibodies. Four percent of cord blood tests were IgG positive for WNV. No cord blood tests were IgM positive for WNV. There were no huge differences between newborn children of seropositive and seronegative mothers concerning any of the development 
parameters or results estimated. Findings suggested that intrauterine WNV infections seemed to be infrequent ${ }^{54}$ and WNV infection during pregnancy did not seem to affect adversely infant health at birth. ${ }^{55}$

Refreshed recommendations for the Evaluation and Testing of Infants with Possible Congenital Zika Virus Infection Testing of newborn children with conceivable inherited Zika infection disease who were born to mothers who traveled to or resided in regions affected by Zika infection during pregnancy ought to be guided by 1) regardless of whether the baby had microcephaly or intracranial calcifications distinguished prenatally or during childbirth and 2) the mother's Zika infection testing results. The assessment of newborn children with microcephaly or intracranial calcifications or babies whose moms have positive or uncertain test results for Zika infection contamination continues as before. Newborn children without microcephaly or intracranial calcifications whose moms have negative Zika infection test results or who were not tried for Zika infection ought to get standard consideration. Assessment and Management of Infants and Children Aged $<18$ Years with Possible Acute Zika Virus Disease, Acute Zika infection sickness ought to be suspected in a baby or youngster matured $<18$ years who ventured out to or lived in an influenced region inside the previous 2 weeks and 2) has at least two of the accompanying indications: fever, rash, conjunctivitis, or arthralgia. Suggested Zika infection lab testing for newborn children and youngsters, prescribed clinical assessment and research center testing for babies with possible innate Zika infection disease, and prescribed long haul line up for babies with possible congenital Zika infection contamination are given in detail. Rules for Breastfeeding for Mothers with Zika Virus Infection, Current proof recommends that the advantages of breastfeeding exceed the hypothetical dangers of Zika infection transmission through breast milk. Prevention of Zika Virus Infection in Infants and Children mosquito nibble prevention include utilizing cooling or window and door screens when indoors, wearing long sleeved shirts and long jeans, utilizing permethrin-treated dress and rigging, and utilizing insect anti-agents. Most Environmental Protection Agency- enlisted insect anti-agents can be utilized to secure youngsters aged $\geq 2$ months against mosquito nibbles. Oil of lemon eucalyptus should not be utilized in kids matured $<3$ Years. ${ }^{56}$ Mosquito mesh can be utilized to cover newborn children in carrier, kid buggies, or bunks to shield them from mosquito nibbles. Data on the protected utilization of bug anti-agents in youngsters is accessible at anti-agents/using insect-anti-agents securely and-viably. ${ }^{57}$ People with Zika infection contamination should find a way to avert mosquito chomps for in any event the main seven day stretch of illness to diminish the hazard for human-to-mosquito-to-human transmission. ${ }^{58}$ December 2013, during the French Polynesian, South Pacific ZIKV outbreak, a 44Years old male in Tahiti presented for symptoms of ZIKV infection of which he had 2 episodes ( 8 weeks apart, each lasting 3 and 7 days, respectively) prior to seeking treatment. He sought treatment 2 weeks after recovering from the second episode after noting signs of hematospermia. RT-PCR was positive for ZIKAV in semen and negative in blood in repeated samples. It is suggested that the replication may have occurred in the genital tract and that transmission via sexual intercourse is possible. ${ }^{59}$

\section{Concluding remarks}

This review was carried out to know more about viral infection. Zika being an airborne flavivirus and having two lineages for its RNA strain so far can be a high risk. To avoid the risks of its association with neuro-retinal or neurological disorders as GBS and Microcephaly prevention methods should be followed. Its transmission through complex means and symptoms confused with DENV and CHIKV should be observed carefully. Serum, saliva, breast milk or semen should be tested through RT-PCR and ELISA for better understanding of risk. More studies should be carried out to develop neutralizing antibodies for any onset of infection or brain damage.

\section{Acknowledgments}

None

\section{Conflicts of interest}

Author declares there is no conflict of interest.

\section{References}

1. Fernandes RL, Cugola L, Russo FB, et al. Zika virus impairs neurogenesis and synaptogenesis pathways in human neural stem cells and neurons. Front Cell Neurosci. 2019;13:64

2. Duffy MR, Chen TH, Hancock WT, et al. Zika virus outbreak on Yap Island, Federated states of Micronesia. N Engl J Med. 2009;360(24):2536-2543.

3. Musso D, Nhan T, Robin E, etal. Potential for Zika virus transmission through blood transfusion demonstrated during an outbreak in French Polynesia, November 2013 to February 2014. Euro surveill. 2014;19(14):20761.

4. Tappe D, Rissland J, Gabriel M, et al. First case of laboratory-confirmed Zika virus infection imported into Europe, November 2013. Euro surveill. 2014;19(4):20685.

5. Mo Y, Alferez Salada BM, Tambyah PA. Zika virus-a review for clinicians. Br Med Bull. 2016;119(1):25-36.

6. Musso D, Gubler DJ. Zika virus. Clin Microbiol Rev. 2016;29(3):487-524.

7. Zammarchi L, Stella G, Mantella A, et al. Zika virus infections imported to Italy: clinical, immunological and virological findings, and public health implications. J Clin Virol. 2015;63:32-35.

8. Bailey AL, Lauck M, Ghai RR, et al. Arteriviruses, pegiviruses, and lentiviruses are common among wild African monkeys. $J$ virol. 2016;90(15):6724-6737.

9. Zanluca C, Melo VC, Mosimann AL, et al. First report of autochthonous transmission of Zika virus in Brazil. Mem Inst Oswaldo Cruz. 2015;110(4):569-572.

10. Brasil P, Calvet GA, Siqueira AM, et al. Zika virus outbreak in Rio de Janeiro, Brazil: clinical characterization, epidemiological and virological aspects. PLoS Negl Trop Dis. 2016;10(4):e0004636.

11. Reyes Y, Bowman NM, Becker-Dreps S, et al. Prolonged Shedding of Zika Virus RNA in Vaginal Secretions, Nicaragua. Emerg Infect Dis. 2019;25(4):808-810. doi:10.3201/eid2504.180977.

12. Lee EM., Titus SA, Xu M., et al. High-Throughput Zika Viral Titer Assay for Rapid Screening of Antiviral Drugs. Assay Drug Dev Technol. 2019;17(3):128-139. doi: 10.1089/adt.2018.881.

13. Christofferson RC. Zika virus emergence and expansion: lessons learned from dengue and chikungunya may not provide all the answers. Am J Trop Med Hyg. 2016;95(1):15-18.

14. Dirlikov E, Ryff KR, Aponte JT, et al. Update: ongoing Zika virus transmission-Puerto Rico, November 1, 2015-April 14, 2016. Morbidity and Mortality Weekly Report(MMWR). 2016;65(17):451-455.

15. Faria NR,Azevedo RDSDS, KraemerMUG, et al. Zika virus in the Americas: early epidemiological and genetic findings. Science. 2016;352(6283):345349.

16. Hahn MB, Eisen RJ, Eisen L, et al. Reported distribution of Ades (stegomyia) Agypti and Ades (stegomyia) albopictus in the United States, 1995-2016 (diptera: culicidA). J Med Entomol. 2016;pii: tjw072. 
17. Hennessey M, Fischer M, Staples JE. Zika virus spreads to new areasregion of the Americas, May 2015-January 2016. MMWR Morb Mortal Wkly Rep. 2016;65(3):55-58.

18. Kindhauser MK, Allen T, Frank V, et al. Zika: the origin and spread of a mosquito-borne virus. Bull World Health Organ. 2016;94(9):675-686C

19. Heukelbach J, Alencar CH, Kelvin AA, et al. Zika virus outbreak in Brazil. J Infect Dev Ctries. 2016;10(2):116-120.

20. Calvet G, Aguiar RS, Melo ASO, et al. Detection and sequencing of Zika virus from amniotic fluid of fetuses with microcephaly in Brazil: a case study. Lancet Infect Dis. 2016;16(6):653-660.

21. Musso D, Cao-Lormeau VM, Gubler DJ. Zika virus: following the path of dengue and chikungunya?. Lancet. 2015;386(9990):243-244.

22. Cauchemez S, Besnard M, Bompard P, et al. Association between Zika virus and microcephaly in French Polynesia, 2013-15: a retrospective study. Lancet. 2016;387(10033):2125-2132.

23. Chibueze EC, Tirado V, Lopes KD, et al. Zika virus infection in pregnancy: a systematic review of disease course and complications. Reprod health. 2017;14(1):28.

24. Driggers RW, Ho CY, Korhonen EM, et al. Zika virus infection with prolonged maternal viremia and fetal brain abnormalities. $N$ Engl $J$ Med. 2016;374(22):2142-2151.

25. Oehler E, Watrin L, Larre P, et al. Zika virus infection complicated by Guillain-Barre syndrome-case report, French Polynesia, December 2013. Euro surveill. 2014;19(9):20720.

26. Cao-Lormeau VM, Blake A, Mons S, et al. Guillain-Barré Syndrome outbreak associated with Zika virus infection in French Polynesia: a casecontrol study. Lancet. 2016;387(10027):1531-1539.

27. Ortega FB, Lavie CJ, Blair SN. Obesity and cardiovascular disease. Circ Res. 2016;118(11):1752-1770.

28. Ventura CV, Maia M, Bravo-Filho V, et al. Zika virus in Brazil and macular atrophy in a child with microcephaly. Lancet. 2016;387(10015):228.

29. Lanciotti RS, Kosoy OL, Laven JJ, et al. Genetic and serologic properties of Zika virus associated with an epidemic, Yap State, Micronesia, 2007. Emerg Infect Dis. 2008;14(8):1232-1239.

30. Hirayama T, Mizuno Y, Takeshita N, et al. Detection of dengue virus genome in urine by real-time reverse transcriptase PCR: a laboratory diagnostic method useful after disappearance of the genome in serum. $J$ Clin Microbiol. 2012;50(6):2047-2052.

31. Gourinat AC, O'Connor O, Calvez E, et al. Detection of Zika virus in urine. Emerg Infect Dis. 2015;21(1):84-86.

32. Musso D, Roche C, Nhan TX, et al. Detection of Zika virus in saliva. J Clin Virol. 2015;68:53-55.

33. Lustig Y, Mendelson E, Paran N, et al. Detection of Zika virus RNA in whole blood of imported Zika virus disease cases up to 2 months after symptom onset, Israel, December 2015 to April 2016. Euro surveill. 2016;21(26).

34. Tsai TF. Congenital arboviral infections: something new, something old. Pediatrics. 2006;117(3):936-939.

35. Zhang X, Xie X, Zou J, et al. Genetic and biochemical characterizations of Zika virus NS2A protein. Emerg Microbes Infect. 2019;8(1):585-602.

36. Hinckley AF, O'Leary DR, Hayes EB. Transmission of West Nile virus through human breast milk seems to be rare. Pediatrics. 2007;119(3):e666e671.

37. Besnard M, Lastere S, Teissier A, et al. Evidence of perinatal transmission of Zika virus, French Polynesia, December 2013 and February 2014. Euro surveill. 2014;19(13):20751.

38. Silasi M, Cardenas I, Kwon JY, et al. Viral infections during pregnancy. Am J Reprod Immunol. 2015;73(3):199-213.
39. Hayes EB. Zika virus outside Africa. Emerg Infect Dis. 2009;15(9):13471350.

40. Bayer A, Lennemann NJ, Ouyang Y, et al. Type III interferons produced by human placental trophoblasts confer protection against Zika virus infection. Cell Host Microbe. 2016;19(5):705-712.

41. Brasil P, Pereira JP Jr, Moreira ME., et al. Zika virus infection in pregnant women in Rio de Janeiro. N Engl J Med. 2016;375(24):2321-2334.

42. Pacheco O, Beltrán M, Nelson CA, et al. Zika virus disease in Colombia - preliminary report. $N$ Engl J Med. 2016;doi: 10.1056/NEJMoa1604037.

43. Franca GV, Schuler-Faccini L, Oliveira WK, et al. Congenital Zika virus syndrome in Brazil: a case series of the first 1501 live births with complete investigation. Lancet. 2016;388(10047):891-897.

44. Martines RB, Bhatnagar J, Keating MK, et al. Notes from the field: evidence of Zika virus infection in brain and placental tissues from two congenitally infected newborns and two fetal losses-Brazil, 2015. Morbidity and Mortality Weekly Report (MMWR). 2016;65(06):159-160.

45. Meaney-Delman D, Hills SL, Williams C, et al. Zika virus infection among US pregnant travelers-August 2015-February 2016. Morbidity and Mortality Weekly Report (MMWR). 2016;65(08):211-214.

46. Oliveira Melo AS, Malinger G, Ximenes R, et al. Zika virus intrauterine infection causes fetal brain abnormality and microcephaly: tip of the iceberg? Ultrasound Obstet Gynecol. 2016;47(1):6-7.

47. de Oliveira WK, Escalante JC, Holanda De Oliveira WTG, et al. Increase in reported prevalence of microcephaly in infants born to women living in areas with confirmed Zika virus transmission during the first trimester of pregnancy-Brazil, 2015. Morbidity and Mortality Weekly Report (MMWR). 2016;65(9):242-247.

48. Teixeira MG, Costa Mda C, de Oliveira WK, et al. The epidemic of Zika virus-related microcephaly in Brazil: detection, control, etiology, and future scenarios. Am J Public Health. 2016;106(4):601-605.

49. Petersen EE, Staples JE, Delman DM, et al. Interim guidelines for pregnant women during a Zika virus outbreak-United States, 2016. Morbidity and Mortality Weekly Report (MMWR). 2016;65(2):30-33.

50. Peterson AT, Osorio J, Qiao $\mathrm{H}$, et al. Zika virus, elevation, and transmission risk. PLoS Curr. 2016;8. pii: ecurrents.outbreaks. a832cf06c4bf89fb2e15cb29d374f9de. doi: 10.1371/currents.outbreaks. a832cf06c4bf89fb2e15cb29d374f9de.

51. Rasmussen SA, Jamieson DJ, Honein MA, et al. Zika virus and birth defect-reviewing the evidence for causality. N Engl J Med. 2016;374(20):19811987.

52. Villamil-Gómez WE, Mendoza-Guete A, Villalobos E, et al. Diagnosis, Management and Follow-up of Pregnant Women with Zika virus infection: A preliminary report of the ZIKERNCOL cohort study on Sincelejo, Colombia. Travel Med Infect Dis. 2016;14(2):155-158.

53. Nguyen Q, Morrow C, Novick L, et al. Intrauterine West Nile Virus infection--New York, 2002. JAMA. 2003;289(3):295-295.

54. Reefhuis J, Gilboa SM, Johansson MA, et al. Projecting month of birth for at-risk infants after Zika virus disease outbreaks. Emerg Infect Diseases. 2016;22(5):828-832.

55. Paisley JE, Hinckley AF, O'Leary DR, et al. West Nile virus infection among pregnant women in a northern Colorado community. 2003 to 2004. Pediatrics. 2006;117(3):814-820.

56. Gaines J, Sotir MJ, Cunningham TJ, et al. Health and safety issues for travelers attending the World Cup and Summer Olympic and Paralympic Games in Brazil, 2014 to 2016. JAMA Intern Med. 2014;174(8):13831390 .

57. Musso D, Roche C, Robin E, et al. Potential sexual transmission of Zika virus. Emerg Infect Dis. 2015c ;21(2):359-361. 
58. Lawrence JH, Sherer ML, Tavlarides-Hontz P, et al. An investigation into the immune response of cultured neural rat cells following Zika virus infection. $J$ Neuroimmunol. 2019;332:73-77. doi: 10.1016/j. jneuroim.2019.04.002. Epub 2019 Apr 3.
59. Fleming-Dutra KE, Nelson JM, Marc Fischer MD, et al. Update: interim guidelines for health care providers caring for infants and children with possible Zika virus infection-United States, February 2016. Morbidity and Mortality Weekly Report (MMWR). 2016;65 (7):182-187. 